\title{
L'incompatibilité pollinique chez le clémentinier : observation in vivo de la croissance des tubes polliniques
}

\author{
D Tisne-Agostini, A Orsini \\ INRA, station de recherche agronomique, San Giuliano, 20230 San Nicolao, France
}

(Reçu te 11 décembre 1989; accepté le 27 juin 1990)

\begin{abstract}
Résumé - L'étude comparative du processus de la fécondation chez 3 clones aspermes de clémentinier (Citrus reticulata Blanco) par l'observation de la croissance des tubes polliniques in vivo, a permis de confirmer l'existence d'anomalies, non pas au niveau des relations stigmate-polien, mais au niveau des relations style-tubes polliniques, laissant supposer la présence d'un système d'auto-incompatibilité pollinique de type gamétophytique. L'arrêt des tubes polliniques, rendus visibles par la microscopie en fluoresence, intervient généralement dès le premier tiers du style, bien que certains d'entre eux parviennent jusque dans les tissus ovariens. Ceci permet d'attribuer un caractère partiel au phénomène mis en cause.

De plus l'appréciation, par le biais d'un indice, de la compatibilité intra- et intervariétale des quelques cultivars étudiés laisse apparaître des degrés variables d'affinités.
\end{abstract}

Citrus reticulata Blanco / autofécondation / microscopie en fluorescence / callose / auto-incompatibilité gamétophytique

Summary - Studies on the fertilization process in the clementine: observations on pollen tube growth in the styles. Since seedless clementine (Citrus reticulata Blanco) is supposed not to have a normal fertilization process 3 varieties were studied in regard to the type of incompatibility and their ability in intercrosses.

A comparison was first made between seedless SRA 85 clementine and seeded Wilking mandarin: pollen germination on stigmata (fig 1), rate of pollen tube growth and tube evolution in the styles (fig 2) were studied using fluorescent microscopy. It was shown clearly for autofertilized SRA 85 that pollen tubes were stopped by callose in the first third of style (fig 3).

Crosses between 3 varieties, Corsica 2, Oroval, SRA 85 were then examined and very limited affinity for each other (fig 8). Estimation of affinity was made by a calculated index (IPC) for each cross.

Main IPC values were under a quarter of the optimal index reached for a simulated total compatible cross.

It was concluded that the normal fertilization process cannot take place in the seedless clementine because of a gametophytic incompatibility. Nevertheless, it is only partial; some flowers can be fertilized with success at a certain stage, to be defined by further studies.

Citrus reticulata Blanco / fluorescent microscopy / self-fertilization / callose / gametophytic incompatibility

\section{INTRODUCTION}

Pour l'amélioration génétique des agrumes, deux techniques classiques de création variétale peuvent être utilisées : la mutagenèse et l'hybridation. Cette dernière, à la différence de la mutation induite, rend obligatoire le passage par la reproduction sexuée. Or ceci n'est pas sans poser de problèmes chez le clémentinier
(Citrus reticulata Blanco) pour lequel un fort degré d'aspermie, en condition d'autopollinisation (Coste et Gagnard, 1956; Chapot, 1963; Garcia-Papi et Garcia-Martinez, 1984) laisse supposer que le processus de la reproduction sexuée ne peut s'effectuer normalement.

Les connaissances acquises sur la qualité des pollens chez le clémentinier (Lacarelle et Miedzyrzecki, 1936; Pereau-Leroy, 1950; 
Damigella et Squilacci, 1959; Orsini, 1988) permettent d'affirmer que l'inaptitude à l'autofécondation n'est pas due à une stérilité mâle. On peut donc présumer qu'il existe des anomalies dans le déroulement de la fécondation, prise au sens large du terme.

Ton et Krezdorn (1966), grâce à une étude in vivo de pistils pollinisés, ont pu montrer que les grains de pollen s'hydrataient et germaient normalement sur la surface stigmatique, mais qu'en revanche, la progression des tubes polliniques à l'intérieur des tissus stylaires se faisait difficilement. Ils avancèrent l'hypothèse selon laquelle il existerait chez le clémentinier, comme chez d'autres espèces de Citrus, un système d'incompatibilité partielle de type gamétophytique.

La vérification d'un tel système d'incompatibilité et la recherche de techniques permettant d'effectuer des hybridations entre clémentiniers nous ont conduit à décomposer l'étude en deux parties, répondant chacune à une préoccupation particulière :

- l'observation in vivo du déroulement du processus de la fécondation, par le biais d'une étude comparative permettant d'identifier les niveaux de blocages caractéristiques du type d'incompatibilité;

- la caractérisation du comportement en autofécondation de certains cultivars et l'appréciation de leurs aptitudes aux croisements, en vue d'hybridations potentielles.

\section{MATÉRIEL ET MÉTHODES}

La première partie de l'étude a été réalisée à partir d'autopollinisations et de croisements réciproques du clémentinier SRA 85, clone asperme, et du mandarinier Wilking 112, dont les fruits ont de nombreux pépins en verger isolé.

La seconde partie porte sur les clones de clémentiniers aspermes, SRA 85, Corsica 2 et Oroval, autopollinisés et croisés entre-eux.

\section{Matériel}

Dans les deux études, 30 fleurs ont été retenues pour chaque traitement. Les autres fleurs des rameaux porteurs ont été éliminées, et ces derniers ont été isolés par des manchons protecteurs. Les fleurs ont été castrées au stade gros bouton blanc, juste avant lanthèse.

Le pollen utilisé a été obtenu par déhiscence des anthères, provoquée artificiellement en chambre chaude à $25^{\circ} \mathrm{C}$. Sa viabilité a été vérifiée par un test FCR (réaction fluorochromatique) avant pollinisation.

Les pistils prélevés sont préparés selon la méthode de Martin citée par Dumas et Knox (1983) : fixation des pistils fendus longitudinalement pendant $24 \mathrm{~h}$ au minimum dans le FAA (mélange 5/5/90 de formol, acide acétique et alcool éthylique à $70^{\circ}$ ); rinçages répétés des coupes à l'eau permutée pour éliminer le fixateur; blanchiment et ramollissement dans une solution de disulfite de sodium à $5 \%$, à $121^{\circ} \mathrm{C}$ pendant 20 min; lavages à l'eau permutée; coloration dans une solution de $\mathrm{K}_{3} \mathrm{PO}_{4} 0,1 \mathrm{M}$ et de bleu d'aniline (Merck) à $0,1 \%$.

Les pistils fixés peuvent être conservés avant traitement à $4^{\circ} \mathrm{C}$ dans de l'éthanol à $70^{\circ}$ pendant quelques semaines. Les échantillons ainsi préparés sont déposés entre lames et lamelles, et observés avant et après écrasement.

\section{Méthodes}

Les observations sont faites sous microscope en fluorescence, en lumière réfléchie bleue ou uitra-violette. La callose apparaît sous forme de bouchons dans les tubes polliniques, et dans leur paroi où elle constitue également la lamelle moyenne. Le trajet des tubes polliniques peut alors être suivi puisqu'en présence du fluorochrome, la callose prend, lorsqu'elle est exposée à la lumière fluorescente, une couleur jaune-vert (lumière d'excitation dans le bleu) ou bleue (lumière d'excitation dans l'UV).

Pour la première partie de l'étude, les fleurs ont été prélevées $1,3,5,7$ et 9 jours après pollinisation, alors que pour la seconde partie, un seul prélèvement a été effectué 7 jours après pollinisation, période au terme de laquelle la fécondation est effective pour des combinaisons compatibles.

Au niveau du stigmate, la densité des tubes polliniques a été convertie en notes de 0 à 5 , allant par classes de 10 de l'absence totale (note 0 ) à plus de 40 tubes (note 5).

Afin de pouvoir quantifier l'efficacité du processus de fécondation selon les combinaisons et afin de faciliter par là-même leur comparaison, l'appréciation de la compatibilité intra et inter-variétale a été basée sur la prise en compte simultanée de deux aspects : un aspect qualitatif, traduit par la progression maximale des tubes dans les trois tiers de style; un aspect quantitatif exprimant le nombre de tubes polliniques dans chacun des tiers de style, par l'attribution de notes de 0 à 3.

Ces deux critères ont été regroupés d'une façon synthétique sous la forme d'un Indice Pondéré de Compatibilité (IPC), inspiré de celui qu'avait utilisé Kapusta (1983) :

avec

$$
I P C=\left(\Sigma X_{c} \cdot n_{c} / N\right)\left(L L_{t}\right)
$$

$$
x_{c}=\frac{1 X_{s}+3 X_{m}+6 X_{i}}{X_{s g}} \times 1 / 6
$$


$c:$ une classe de notation de pistil, selon les notes affectées au stigmate et à chaque tiers de style. $X_{s}, X_{m}$, $X_{i}$ : notes accordées aux tiers supérieur, moyen et inférieur de style. Le tiers supérieur est sous-jacent au stigmate tandis que le tiers inférieur est situé immédiatement au-dessus de l'ovaire. $X_{s g}$ : note stigmatique, variant de 1 à 5 , tenant lieu de terme correctif de l'efficience de la pollinisation. Les classes de notes sont les mêmes que celles de l'étude cinétique. $X_{c}$ : note globale de la classe de notation avec affectation d'un poids (coef $1,3,6$ ) à chaque tiers de style. Sa valeur optimale est rapportée à 1 par l'utilisation du coefficient $1 / 6 . n_{c}$ : effectif de pistils appartenant à chaque classe. $N$ : effectif total de pistils observés pour le croisement considéré. $L$ : longueur moyenne des styles pour le croisement considéré. $L_{t}$ : longueur moyenne des styles de l'ensemble des croisements.

\section{RÉSULTATS}

\section{Étude cinétique comparative des processus de la fécondation par observation in vivo de la croissance des tubes polliniques}

\section{Présence des tubes polliniques dans les stigmates}

Le tableau I récapitule les résultats obtenus à partir de 75 observations par traitement.

Les notes moyennes de densité des tubes dans le stigmate sont très voisines de la valeur maximale obtenue avec le croisement clémentinier SRA $85 \times$ mandarinier Wilking. Elles sont
Tableau I. Appréciation de la présence des tubes polliniques dans les stigmates pour les 4 traitements considérés.

\section{Nature du traitement Note stigmatique \\ Moyenne Minimale Maximale}

\begin{tabular}{llll} 
Wilking $\times$ SRA 85 & 4,85 & 0 & 5 \\
Wilking $\times$ Wilking & 4,91 & 0 & 5 \\
SRA 85 × Wilking & 5,00 & 5 & 5 \\
SRA 85 x SRA 85 & 4,75 & 2 & 5 \\
\hline
\end{tabular}

toutes très largement supérieures à 4 , ce qui, selon le système de notation adopté, permet de considérer qu'il y a au moins 40 tubes polliniques par stigmate (souvent même beaucoup plus).

La présence des tubes polliniques dans l'ensemble des stigmates dépend d'une part de la quantité de pollen déposé sur le stigmate et, d'autre part de la faculté des grains de pollen à germer et croître dans celui-ci. Or l'observation a permis de constater que les stigmates ayant reçu les notes les plus faibles étaient également ceux qui avaient peu ou pas de pollen sur la surface stigmatique. II s'avère donc que la note affectée à chaque stigmate dépend beaucoup plus de la quantité de pollen déposé que de la germination et/ou de la croissance des tubes dans les

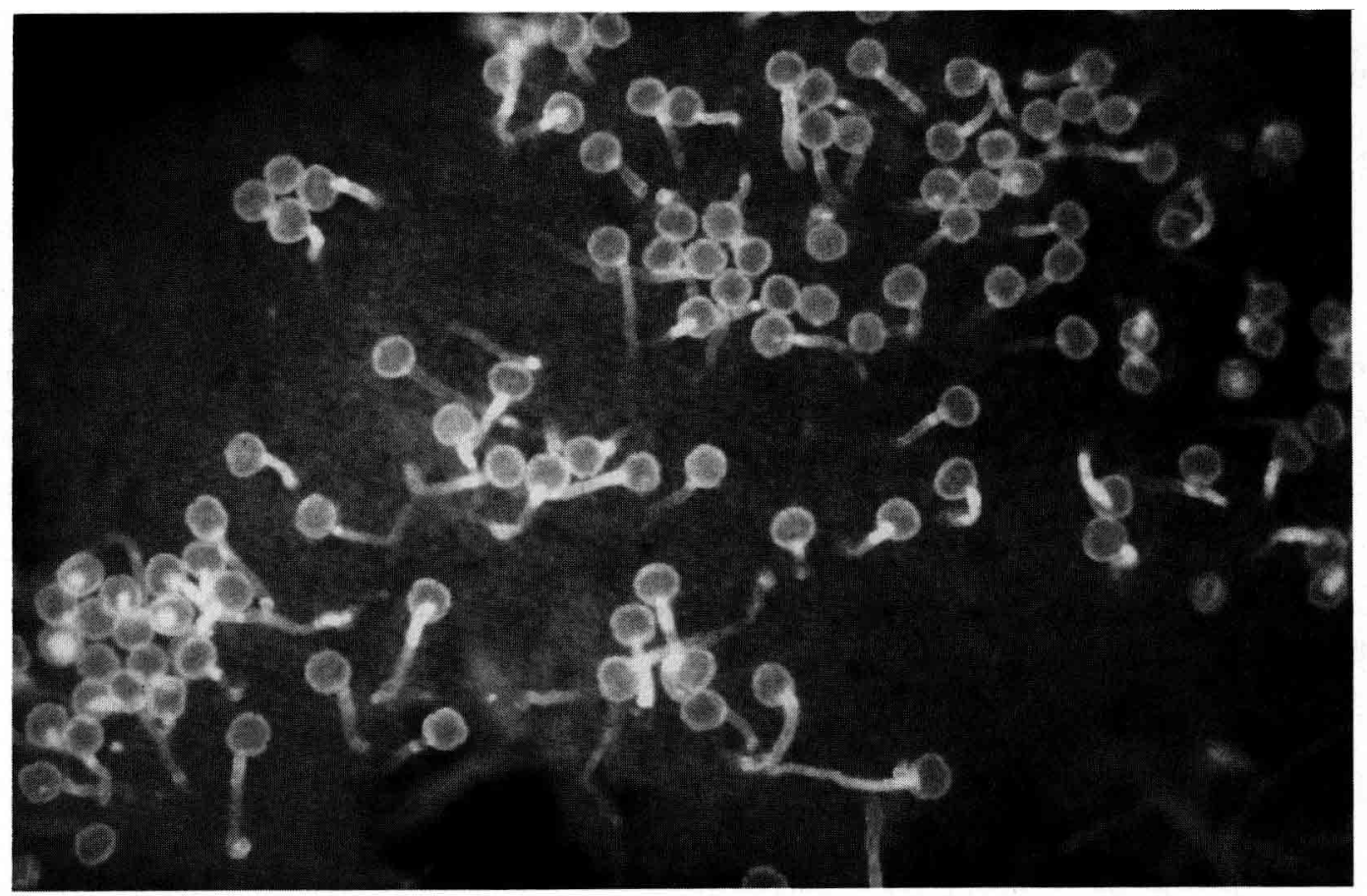

Fig 1. Germination de grains de pollen sur la surface stigmatique chez la clémentine SRA 85 en autopollinisation. 
tissus stigmatiques. Par conséquent, les relations pollen-stigmate sont très similaires d'un traitement à un autre et ne présentent aucune anomalie en situation d'autopollinisation (fig 1).

\section{Évolution des tubes polliniques dans le style et l'ovaire}

\section{Progression des tubes polliniques} dans trois conditions de pollinisation

Les observations de germination en fonction du temps, à partir de 15 fleurs par croisement, sont représentées sur la figure 2 . Celle-ci représente le pourcentage de colonisation par les tubes polliniques, des différentes parties du style et de l'ovaire, en fonction du temps après pollinisation. Trois combinaisons sont mises en comparaison : SRA $85 \times$ SRA 85 , Wilking $\times$ SRA 85, SRA $85 \times$ Wilking.

Deux types de comportement sont observés selon que le clémentinier SRA 85 est autopollinisé ou hybridé avec le mandarinier Wilking.

En autopollinisation, les tubes polliniques de SRA 85 s'arrêtent, pour une large part, dans le tiers supérieur des styles bien que l'on ait pu en observer, dans quelques rares cas, jusque dans les ovaires (fig 3).

En revanche, pour les croisements où SRA 85 est soit parent mâle, soit parent femelle, les ovaires sont atteints dès le $5^{\ominus}$ jour (fig 4).
Si on met en relation les observations au niveau du stigmate et celles du premier tiers de style, pour les trois croisements, on remarque que pour le traitement en autopollinisation les tubes polliniques éprouvent une certaine difficulté à traverser la zone contiguë au stigmate et au style.

\section{Élongation des tubes polliniques}

Les mesures ont été effectuées sur les cinq tubes les plus longs afin de quantifier la progression maximale des tubes à l'intérieur des organes femelles.

La nécessité de faire un rapprochement entre les longueurs de tubes et celles des différentes parties du pistil, nous a conduits à établir des comparaisons par couples de traitements ayant le même parent femelle, et par conséquent des longueurs de styles semblables.

De la figure 5, il semble se dégager un processus normal d'élongation des tubes polliniques au vu de l'allure identique des courbes présentées par les trois traitements Wilking $X$ Wilking, SRA $85 \times$ Wilking, Wilking $X$ SRA 85 : les tubes ont une croissance régulière de sorte qu'ils atteignent l'ovaire avant le $5^{\mathrm{e}}$ jour suivant la pollinisation (fig 6).

Seule l'autopollinisation de SRA 85 présente une cinétique très différente; on observe un palier à partir du $3^{e}$ jour traduisant un arrêt de la croissance au niveau du premier tiers de style.

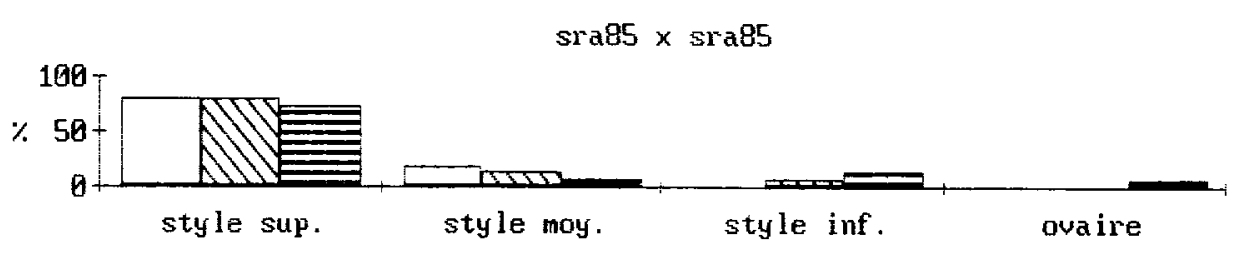

wilking $x$ sra85
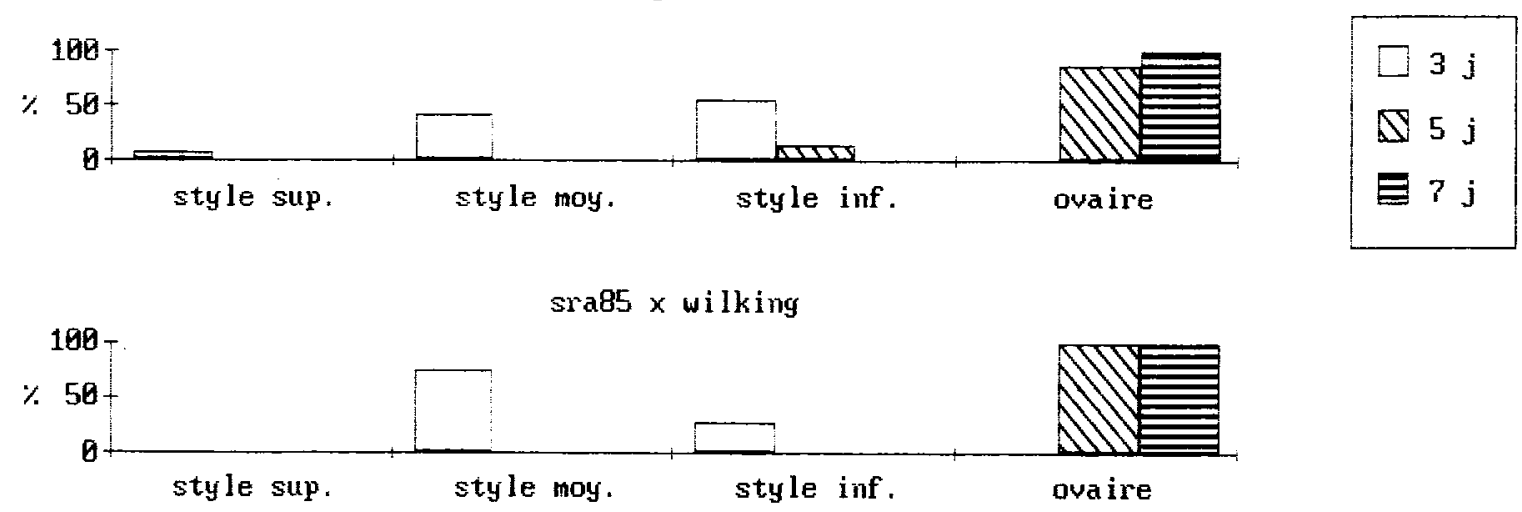

Fig 2. Localisation des tubes polliniques exprimée en pourcentage des différentes parties du style et des ovaires atteints, en fonction du temps après pollinisation et pour trois des croisements étudiés. 


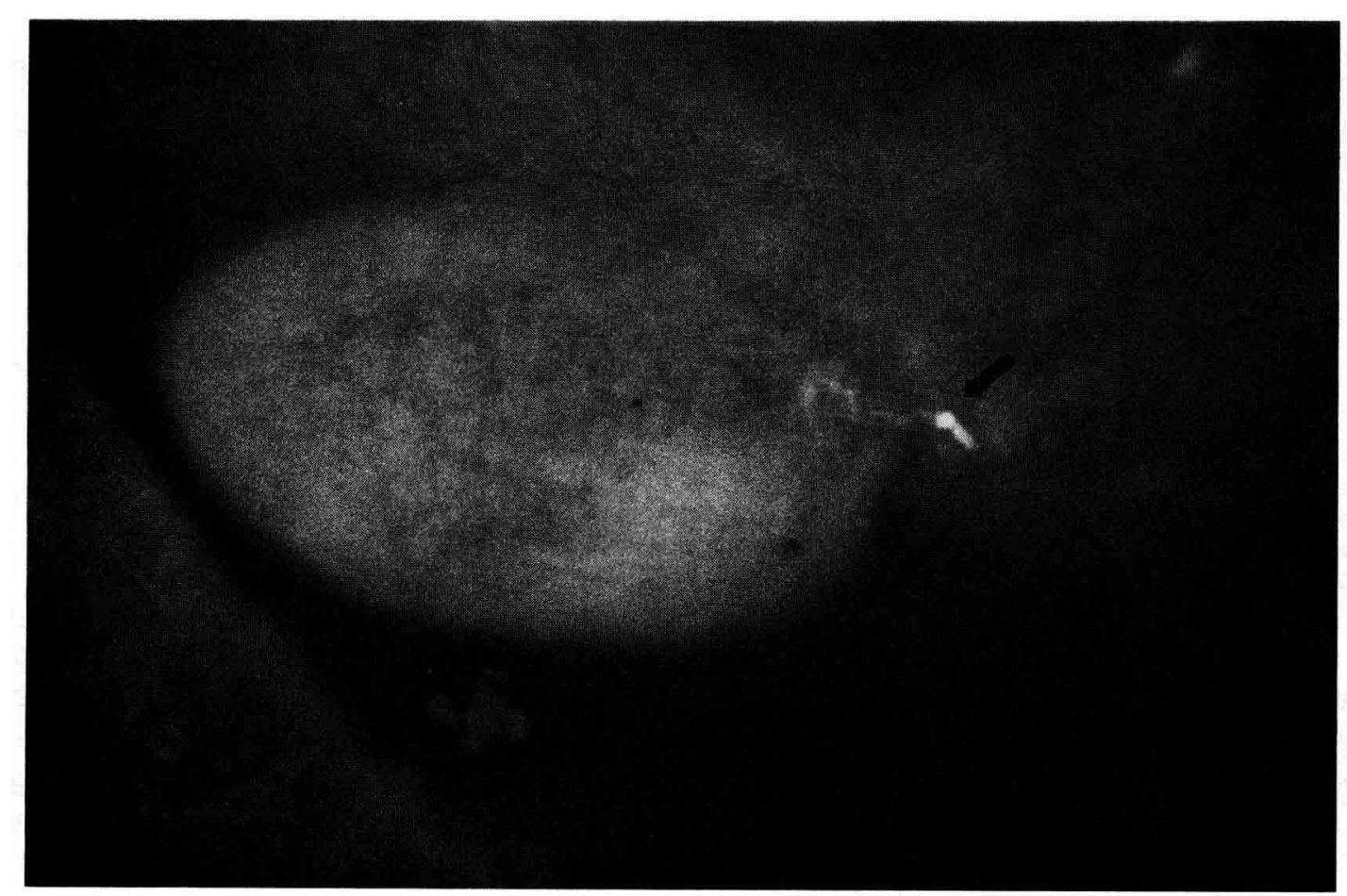

Fig 3. Tube polinique de clémentine SRA 85 pénétrant dans un ovule du même clone.

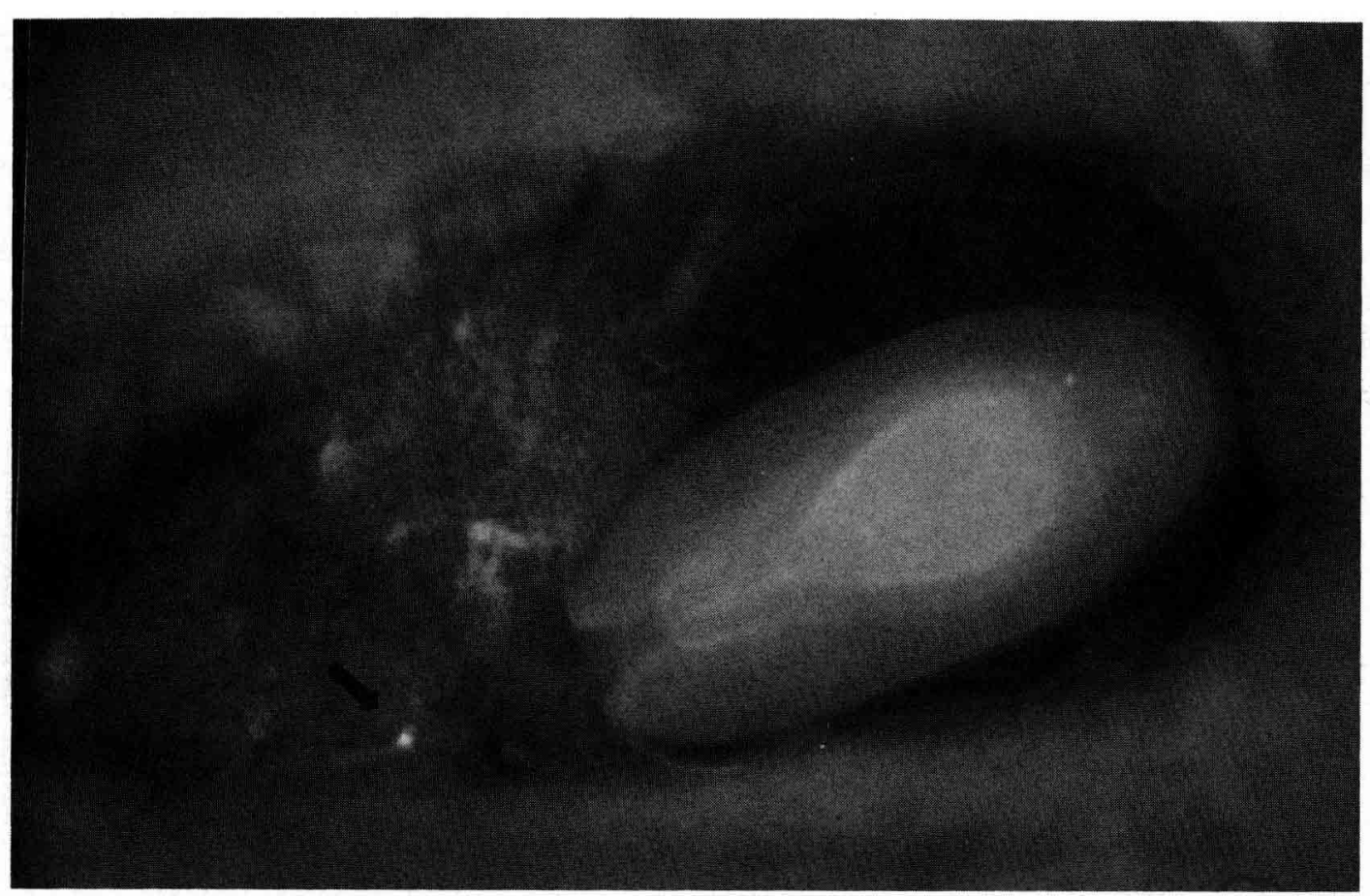

Fig 4. Tubes polliniques de mandarine Wilking à proximité d'un ovule de clémentine SRA 85, 5 jours après pollinisation.

\section{Appréciation de la compatibilité intra et intervariétale}

Les observations réalisées 7 jours après pollinisation, à partir de 30 pistils pour chaque croisement, et exprimées sur la figure 7, montrent, pour trois combinaisons, que des tubes polliniques peuvent parvenir à la base du style avec un taux de réussite variable selon le croisement considéré. En revanche, pour les trois autres traitements, on observe un blocage des tubes dans le premier tiers de style, de telle sorte qu'ils n'atteignent que rarement le tiers moyen et pour ainsi dire jamais, le tiers inférieur.

La figure 8 représente les valeurs de I'IPC, calculé pour chaque croisement, 7 jours après 


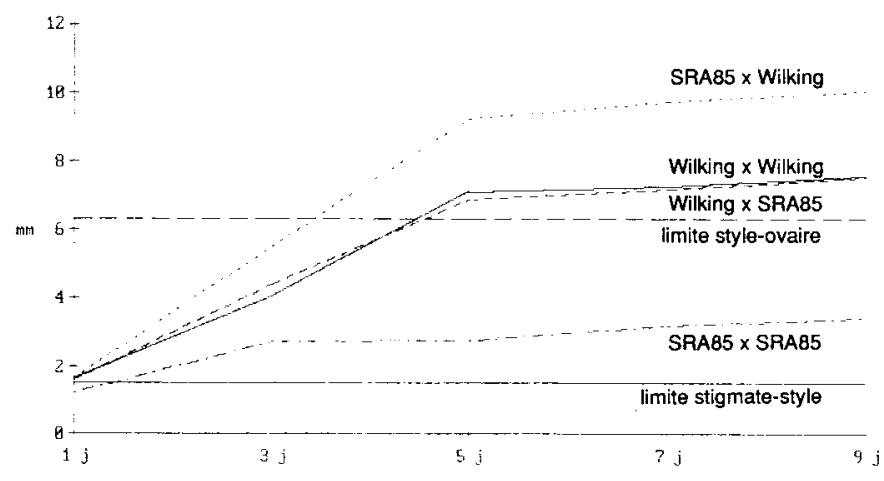

Fig 5. Longueur moyenne de pistil parcourue par les 5 tubes polliniques les plus longs au cours du temps, pour les quatre croisements étudiés.

pollinisation, à partir de 30 observations par croisement. Un croisement fictif totalement compatible, pour lequel l'indice est optimal, a été ajouté comme référence.

Dans l'ensemble, les cultivars présentent un faible degré de compatibilité puisque la majorité des IPC ne dépasse pas le quart de la valeur de l'indice optimal.

Deux groupes de compatibilité peuvent être distingués, mais il n'est cependant pas possible d'expliquer les différences observées par la seule existence de degrés variables d'affinité entre les cultivars. En effet, il est à noter que les deux croisements investis des meilleurs indices, sont également ceux pour lesquels la pollinisation a été réalisée plus tardivement, les condi- tions climatiques (température) étant néanmoins identiques.

\section{Groupe 1 : Corsica $\times$ Corsica 2 SRA $85 \times$ Corsica 2 SRA $85 \times$ Oroval \\ Groupe 2 : Corsica $2 \times$ Oroval SRA $85 \times$ SRA 85 Corsica $2 \times$ SRA 85}

\section{DISCUSSION}

Le comportement des tubes polliniques in vivo, apprécié par la technique de la microscopie en fluorescence, diffère selon le niveau du pistil.

Dans le stigmate, l'hydratation, la germination et la croissance des tubes s'opèrent d'une façon normale et analogue chez le mandarinier Wilking et chez le clémentinier SRA 85, quel que soit le type de croisement.

En revanche, c'est au niveau du style que se distinguent les croisements. Lorsque SRA 85 est autopollinisé, les accumulations de callose sont importantes au niveau du premier tiers de style, définissant ainsi une zone d'interférence (Battacharjya et Linksen, 1955) où la plupart des tubes polliniques sont arrêtés. Cela confirme l'existence de problèmes au niveau des relations style-pollen, laissant supposer fortement une auto-incompatibilité de type gamétophytique.

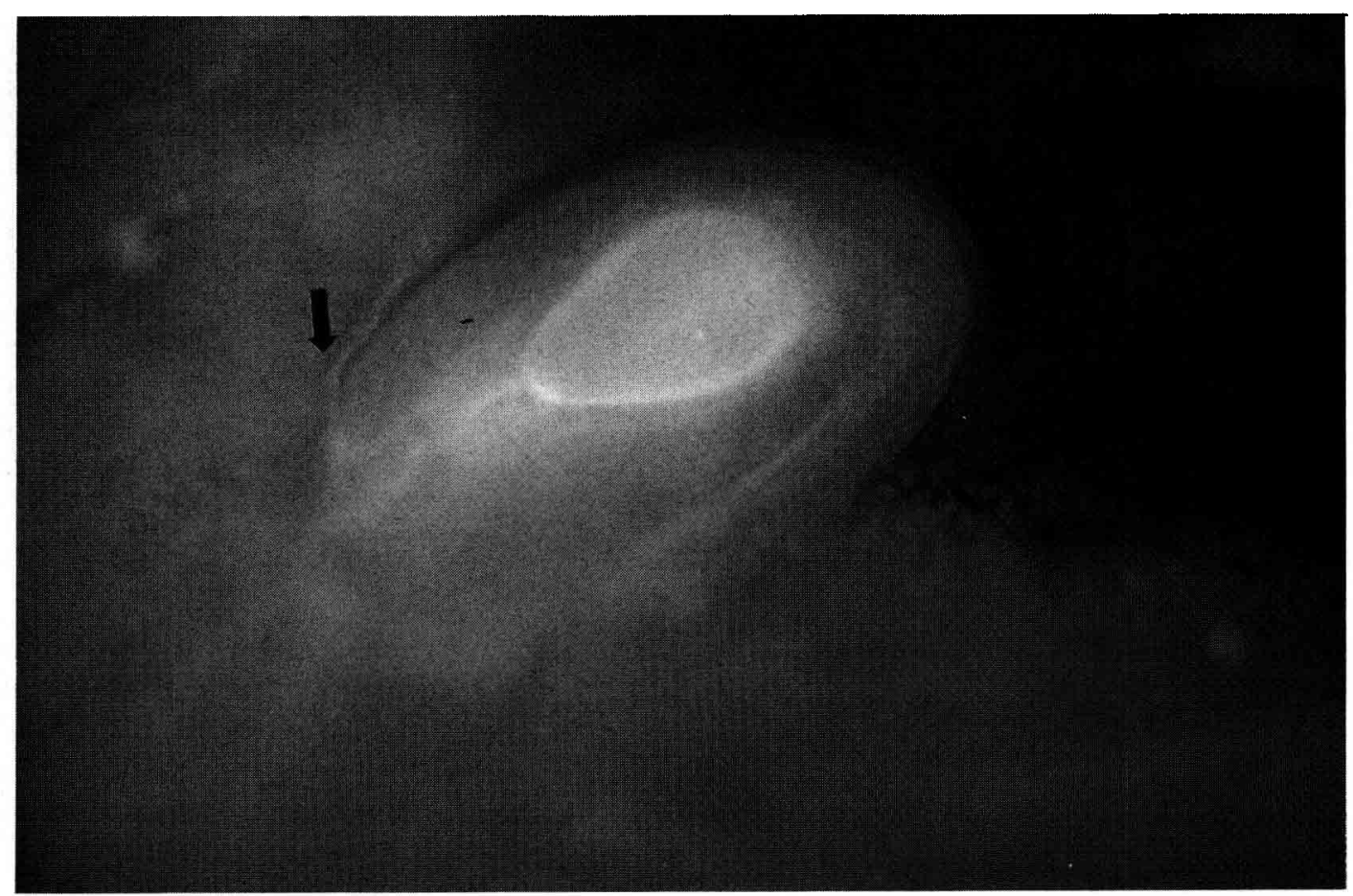

Fig 6. Tube pollinique de mandarine Wilking pénétrant dans un ovule de clémentine SRA 85, 5 jours après pollinisation. 


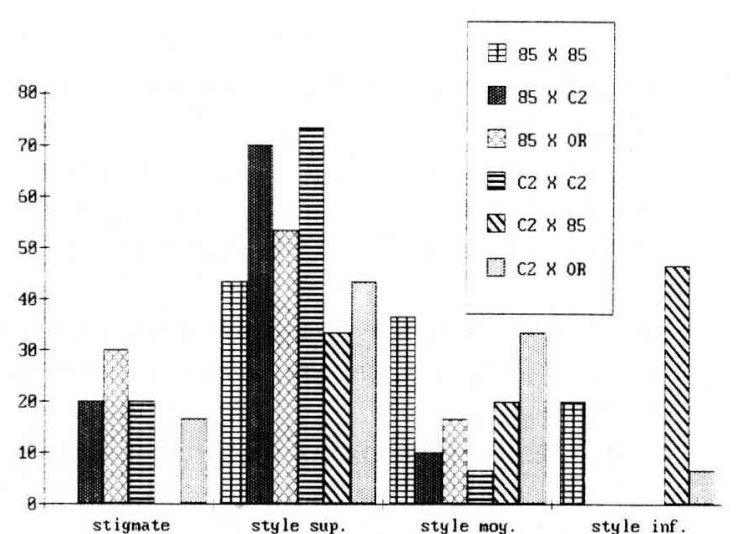

Fig 7. Localisation des tubes poiliniques exprimée en pourcentage des stigmates et des différentes parties du style atteints 7 jours après pollinisation, pour les six croisements étudiés entre SRA 85, Corsica 2 et Oroval.

La mise en évidence du même type d'incompatibilité chez plusieurs cultivars de clémentinier, suggère que celle-ci est de nature génétique. De plus l'existence d'un locus $S$ chez certaines espèces du genre Citrus (Soost, 1969) autorise à émettre l'hypothèse que le clémentinier est soumis à un déterminisme analogue (théorie de EAST des allélomorphes multiples, citée par Pereau-Leroy, 1950).

Sur de telles bases génétiques, deux théories peuvent être retenues :

- l'une décrite par Lewis (1949), selon laquelle l'expression de l'incompatibilité repose sur un mécanisme oppositionnel où la confrontation d'allèles identiques au locus $S$ (pollen et style) entrainerait l'initiation ou la synthèse de molécules inhibitrices s'opposant à la progression des tubes polliniques;

- l'autre formulée par Kroes (1973) selon laquelle l'expression du phénomène est basée sur un mécanisme complémentaire où la présence concomitante de deux allèles différents se traduirait par l'activation de substances ou de stimuli nécessaires à la croissance des tubes polliniques.

Or, l'étude menée nous a permis de distinguer deux types de réactions morphologiques d'arrêt des tubes polliniques, que l'on pourrait associer respectivement aux deux modèles précités :

- soit une importante masse callosique se met en place à l'extrémité des tubes qui adoptent alors des tracés sinueux dans les tissus du premier tiers de style;

- soit on observe un effilement des tubes, comme si ceux-ci semblaient manquer de vigueur pour atteindre l'ovaire. Ce comportement des tubes polliniques semble étayer l'hypothèse de Kakizaki, retenue par Pereau-Leroy (1950) dans le cas précis de clémentinier, admettant

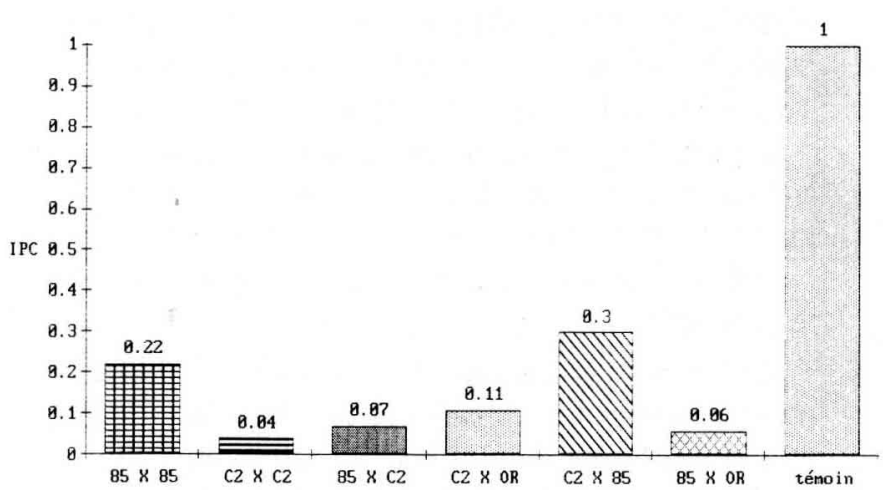

Fig 8. IPC calculé à partir de pistils prélevés 7 jours après pollinisation, pour les six croisements étudiés.

l'association à la série d'allélomorphes $S$ d'une série de facteurs favorables à la croissance des tubes.

Par ailleurs, il semble que l'autoincompatibilité ne revête qu'un caractère partiel puisque l'étude in vivo a permis d'observer des tubes polliniques atteignant l'ovaire dans quelques cas d'autopollinisations de SRA 85. De même, dans quelques fruits issus des mêmes auto-pollinisations, on a pu dénombrer un nombre de pépins sensiblement égal à celui observé dans des fruits issus de croisements compatibles (Wilking $\times$ Wilking, Wilking $\times$ SRA 85).

La fécondation peut donc avoir lieu sous certaines conditions, dont l'une pourrait être liée au stade de pollinisation (fleur au stade bouton ou fleur épanouie). Selon certains auteurs (PereauLeroy, 1950; Soost, 1956; Garcia-Papi et Garcia-Martinez, 1984) les pollinisations de fleurs à un stade pré-anthèse permettraient la fécondation car le tube pollinique, ayant un parcours moins long à effectuer (styles plus courts chez des fleurs au stade bouton qu'au stade fleur épanouie), parviendrait à l'ovaire avant la mise en place d'un système d'inhibition. Or, dans la présente étude, les pollinisations ont été réalisées sur des fleurs en boutons, c'est-à-dire un peu avant l'anthèse. L'expression de l'incompatibilité pouvant évoluer rapidement à l'approche de l'anthèse, notamment si les conditions ambiantes sont favorables, certaines pollinisations ont pu conduire à fécondation. Ceci pourrait expliquer, au moins partiellement la présence de tubes polliniques dans quelques ovaires, et de pépins dans certains fruits.

D'autre part, l'appréciation du degré d'incompatibilité a permis de déceler des différences 
entre croisements. Même si l'on suppose que les cultivars peuvent avoir des combinaisons alléliques différentes pour le gène $S$, leur conférant ainsi des degrés d'affinité variables, il nous paraît plus probable d'invoquer là-aussi l'importance du stade de la fleur au moment de la pollinisation comme facteur de réussite de la fécondation.

Outre d'avoir permis de préciser le déroulement de certaines étapes essentielles du processus de la reproduction, cette étude apporte la confirmation que le clémentinier est gouverné par un système d'auto-incompatibilité gamétophytique partielle dont le mécanisme et le déterminisme demeurent hypothétiques.

Cependant, on peut utiliser l'incidence du stade physiologique de la fleur pollinisée sur l'expression de l'incompatibilité comme moyen de contourner les barrières aux auto-fécondations ou aux croisements entre cultivars incompatibles de clémentinier.

\section{RÉFÉRENCES}

Battacharjya SS, Linksen HF (1955) Recent advances in the physiology of the self-sterility in plants. Sci Cult 20, 8, 370-373

Chapot H (1963) Clémentine avec ou sans pépins. Fruits (Paris) 18, 259-261

Coste A, Gagnard J (1956) Étude sur la pollinisation chez le clémentinier. Livre du IVe Congrès Interna- tional de l'Agrumiculture Méditerranéenne. Tel-Aviv, Israël, 277-287

Damigella P, Squilacci A (1959) Estudio sulla biologia fiorale del Clementine e prove di messa o frutto di piante improductive. Tec Agric (Catania) 4-5, 440462

Dumas C, Knox RB (1983) Callose and determination of pistil viability and incompatibility. Theor App/ Genet 67, 1-10

Garcia-Papi MA, Garcia-Martinez JL (1984) Fruit set and development in seeded and seedless clementine mandarin. Sci Hortic (Amst) 22, 113-119

Kapusta V (1983) Levée de l'auto-incompatibilité chez le pommier. Étude comparative de clones autocompatibles. Mémoire ENSA Rennes, $32 \mathrm{p}$

Kroes HW (1973) An enzyme theory of selfincompatibility. Incompatibility news' letter 2, 5-14

Lacarelle A, Miedzyrzecki $\mathrm{CH}$ (1936) Contribution à l'étude du clémentinier au Maroc. La Terre Marocaine, 19-25

Lewis D (1949) Incompatibility in flowering plants. Biol Rev 24, 472-496

Orsini A (1988) Contribution à l'étude de l'inaptitude à la fécondation de quelques cultivars de clémentinier sans pépins. Mém ENITH Angers, $47 \mathrm{p}$

Pereau-Leroy $P$ (1950) Observations sur le pollen des agrumes. Fruits d'Outre-Mer 5, 8, 288-293

Soost RK (1956) Unfruitfullness in the Clementine mandarin. Proc Am Soc Hortic Sci67, 171-175

Soost RK (1969) The incompatibility gene system in Citrus. Proc First Int Citrus Symposium 1, 189-190

Ton LD, Krezdorn AH (1966) Growth of pollen tubes in three incompatible varieties of citrus. Proc Am Soc Hort Sci 89, 211-215 